\title{
The structure of frontoparallel haptic space is task dependent
}

\author{
FROUKE HERMENS \\ Radboud University Nijmegen, Nijmegen, The Netherlands \\ ASTRID M. L. KAPPERS \\ Helmholtz Institute, Utrecht University, Utrecht, The Netherlands \\ and \\ STAN C. A. M. GIELEN \\ Radboud University Nijmegen, Nijmegen, The Netherlands
}

\begin{abstract}
In three experiments, we investigated the structure of frontoparallel haptic space. In the first experiment, we asked blindfolded participants to rotate a matching bar so that it felt parallel to the reference bar; the bars could be at various positions in the frontoparallel plane. Large systematic errors were observed, in which orientations that were perceived to be parallel were not physically parallel. In two subsequent experiments, we investigated the origin of these errors. In Experiment 2, we asked participants to verbally report the orientation of haptically presented bars. In this task, participants made errors that were considerably smaller than those made in Experiment 1. In Experiment 3, we asked participants to set bars in a verbally instructed orientation, and they also made errors significantly smaller than those observed in Experiment 1. The data suggest that the errors in the matching task originate from the transfer of the reference orientation to the matching-bar position.
\end{abstract}

Several studies have aimed at determining the relationship between the structure of perceived visual space and Euclidean physical space (for an overview, see Wagner, 1985). In a Euclidean space, two parallel lines remain parallel when one (or both) of them is translated. However, Helmholtz (1867/1962) showed that wires arranged by a participant in a perceived frontoparallel plane do not lie in a physically frontoparallel plane. Moreover, Hillebrand (1902) found that two lines that appear to be equidistant in depth are not physically equidistant. These results have led to the suggestion that visually perceived space is distorted and not Euclidean, and they led Luneburg (1947) to conclude that visually perceived space is represented better by a curved Riemannian space. Specifically, Luneburg suggested that perceived space has a Riemannian structure with a negative curvature.

In the past decades, this assumption has been tested via studies in which the structure of visual space was investigated using a number of experimental methods, including distance matching (Meng \& Sedgwick, 2001), orientation matching (Cuijpers, Kappers, \& Koenderink, 2002), triangulation (Fukusima, Loomis, \& Da Silva, 1997), pointing (Koenderink, van Doorn, Kappers, \& Lappin, 2002), and

We thank the Netherlands Organization for Scientific Research (NWO) for financial support and Hans Kolijn for building the setup. Correspondence relating to this article may be sent to S. C. A. M. Gielen, Geert Grootteplein 21, 6525 EZ Nijmegen, The Netherlands (e-mail: s.gielen@science.ru.nl). direct testing of axioms (Koenderink, van Doorn, Kappers, \& Todd, 2002; Todd, Oomes, Koenderink, \& Kappers, 2001). The perceived relative orientation of two physically parallel bars at various positions in the visual field depends on the nature of the visually perceived space. For visual and haptic orientation matching tasks, Cuijpers, Kappers, and Koenderink (2003) quantitatively compared experimental data and predictions based on a Euclidean geometry or a Riemannian geometry with a positive or negative curvature. They found that neither model incorporated the experimental observations correctly, but that zero curvature yielded the best fit of the data. In addition, they showed that for other tasks, such as the pointing task, a Riemannian space did not yield an appropriate fit.

These investigations of visual space have been extended to the haptic modality. Blumenfeld (1937) asked participants to hold two strings parallel to each other that at one end were attached to a table at points equidistant from the median plane. The results were far from veridical: The lines produced diverged toward the participant as long as the distance between the lines was less than the distance between the shoulder joints; beyond this distance, the lines gradually became parallel, and for some of the participants they even converged.

Much evidence in the literature supports the existence of haptic oblique effects; that is, performance in certain tasks is worse when the orientation of the stimuli is (or should be made) oblique than when the stimuli are either horizontal or vertical (see, e.g., Appelle \& Countryman, 1986; Appelle \& Gravetter, 1985; Gentaz \& Hatwell, 
1995, 1999; Lechelt, Eliuk, \& Tanne, 1976; Lechelt \& Verenka, 1980). Traditionally, an oblique effect has appeared as a reduction in the accuracy of the settings (that is, an increase in the standard deviation of repeated settings). Later studies have also investigated the oblique effect on systematic errors (e.g., Kappers, 2003).

These studies of the haptic oblique effect have shown that several factors affect its size. The effect has been larger when participants performed the matching task using both hands (bimanual matching) than when they used the same hand for inspection and matching (unimanual matching; see Appelle \& Countryman, 1986; Gentaz \& Hatwell, 1995). Variability in the settings has typically decreased when participants performed the matching after a delay between inspection and matching (Lechelt \& Verenka, 1980). Certain tasks during the delay, such as recalling the letters of the alphabet in reverse order, have also affected the variability of settings (Gentaz \& Hatwell, 1999). In addition, variability in matching has depended on the orientation of the plane (horizontal, frontal, or sagittal) in which the matching was performed (Gentaz \& Hatwell, 1995, 1996). The effect of the matching plane is thought to be due to gravitational cues (see also Luyat, Gentaz, Corte, \& Guerraz, 2001), but it might also relate to forearm orientation (Kappers, 2003; Soechting \& Flanders, 1993). Gentaz and Hatwell $(1996,1999)$ showed that errors were different in conditions in which participants could rest their arms on the table than in conditions in which they were asked to hold their arms just above the table surface.

These oblique-effect experiments were followed by a series of haptic-space studies (Kappers, 1999, 2002, 2003; Kappers \& Koenderink, 1999; Newport, Rabb, \& Jackson, 2002; Zuidhoek, Kappers, \& Postma, 2005; Zuidhoek, Kappers, van der Lubbe, \& Postma, 2003). In these experiments, the focus was on determining the structure of the internal representation of space on the basis of haptic perception of object orientations. The difference in research questions between these studies and those of the oblique effect has led to differences in both data collection and data analysis. One difference has involved the number of locations at which the stimuli were presented: Most of the haptic-space studies have focused on stimuli presented at several positions within a large part of the workspace, but in oblique-effect studies stimuli have often been presented at a few (generally one or two) positions only. Another difference has related to the types of errors that were studied: The oblique-effect studies have focused on variability in the settings, and the hapticspace studies have investigated systematic errors.

In their haptic-space study, Kappers and Koenderink (1999) investigated the structure of horizontal haptic space by presenting the reference bar at one of nine positions located on a $3 \times 3$ grid. Orientation matching performance was measured for each of the remaining eight positions on the grid. These extensive measurements resulted in vector plots showing the structure of the horizontal haptic space. These vector plots showed that the size of matching errors increased with the horizontal distance between the two bars. In their study, Kappers and Koenderink presented all stimuli to the right of participants and asked them to use only the right hand to match the orientations of the bars. Kappers (1999) extended their study by looking at bimanual matching across a larger portion of horizontal space and found large, systematic deviations from physical parallelity that increased with the horizontal distance between the bars. These errors were larger for bimanual than for unimanual matching. Kappers (2002) investigated the midsagittal plane. Systematic errors were likewise found for this plane, and they were also larger for bimanual matching. The findings by Kappers (2002) extend earlier measurements of the oblique effect in the sagittal plane, in which stimuli at just two locations were presented (Gentaz \& Hatwell, 1995).

Kappers $(2003,2004)$ concluded that the results are consistent with the idea that parallelity judgments are made in a frame of reference intermediate between one that is fixed in space (an allocentric reference frame) and an egocentric reference frame, most likely one fixed to the hand. In an allocentric reference frame, physically parallel bars would haptically also be perceived as parallel; in an egocentric reference frame fixed to the hand, however, haptically parallel would mean "the same orientation with respect to the hand." As the horizontal distance between the reference and test bars becomes larger, the difference in the orientations of the two hands becomes larger, and the deviation becomes larger as well. Participants with large deviations give relatively more weight to the contribution of the egocentric reference frame. In the experimental conditions of Kappers (2003, 2004), hand orientation was oblique with respect to the setup, and as a consequence oblique reference orientations were actually aligned with or perpendicular to the hand, and thus were nonoblique. In these conditions, an oblique effect in an egocentric reference frame would show as a reverse oblique effect in the experimental results, and that is what was found: Participants with large mean errors had larger matching errors for horizontal and vertical orientations than for oblique orientations.

The present experiments were designed to answer two research questions. First, we were interested to see whether haptic matching errors could be observed for the frontoparallel plane and whether these errors would resemble those found for the horizontal plane (Kappers, 1999; Kappers \& Koenderink, 1999) and the sagittal plane (Kappers, 2002). An investigation of the structure of frontoparallel haptic space would allow a future comparison of the three orthogonal haptic spaces (horizontal, frontoparallel, and sagittal) with the structure of 3-D haptic space. Second, our experiments were meant to determine whether similar information about the structure of haptic space could be obtained using different tasks. The three tasks we used were orientation matching, orientation naming, and instructed orientation production. We found that these different tasks did not provide converging evidence for a 
unique structure of haptic space; instead, a combination of two spaces, one allocentric, one egocentric, provided a better account of the data.

\section{EXPERIMENT 1}

In Experiment 1, we investigated the structure of frontoparallel space by asking participants to match the orientation of a reference bar to that of a matching bar. This matching task has been used before to investigate the structure of horizontal haptic (Kappers, 1999; Kappers \& Koenderink, 1999) and visual spaces and to investigate sagittal haptic space (Kappers, 2002). We restricted the number of bar positions to four. By comparing the errors for these four positions within three different tasks (Experiments 1-3), a good first impression of frontoparallel space could be obtained.

Experiment 1 included an investigation of the upper arm, forearm, and hand orientations in the matching task on a trial-by-trial basis. Mean matching errors have been shown to be related to hand orientation during matching of the orientations of the two bars (Kappers, 2003). In an additional session, we video-recorded 6 participants while they performed the matching task. From these video recordings were extracted the frames at the beginning (the first time at which the bars were touched) and the end (just before releasing the bars) of the movements. By localizing the positions of shoulder, elbow, wrist, and fingertip in these frames, we could both estimate the orientations of the upper arm, forearm, and hand and investigate the relation of these orientations with the matching errors.

\section{Method}

Participants. Fifteen participants ( 7 male) took part in the experiment. We tested the handedness of the participants using Coren's test (Coren, 1993), and all except 1 (G.A.) were right handed. The left-handed participant received the same instructions as the righthanded participants. Seven of the participants were naive with respect to the experimental setup and the haptic matching task. These participants were paid for their participation. The remaining participants had taken part in one or more haptic matching experiments for the horizontal plane. No participant had been involved before in a haptic matching experiment for the frontoparallel plane.

Six of the right-handed participants took part in an additional session in which we video-recorded their movements and arm postures during matching. Four of these participants also took part in the matching task without video-recording.

Apparatus. For the haptic matching task, two metal bars were used. These bars were attached to a vertically positioned whiteboard in the frontoparallel plane. Each bar was $20 \mathrm{~cm}$ in length and $1 \mathrm{~cm}$ in diameter. Each bar could be rotated around a pin attached to its center, which fit into holes in the whiteboard. The bars were held in place by two magnets and could be placed on each of the four corners of a $4 \times 4$ grid measuring $90 \times 90 \mathrm{~cm}$. All bar positions were at a distance of $45 \mathrm{~cm}$ from the body midline. The top two bar positions were at a distance of $30 \mathrm{~cm}$ from the shoulders, and the bottom two bar positions were at a distance of $60 \mathrm{~cm}$ from the shoulders. A sheet attached to the whiteboard showed a $360^{\circ}$ protractor around each possible bar position; these protractors were used to measure the orientation of the bars, which could be done with an accuracy of $1^{\circ}$. Figures $1 \mathrm{~A}$ and $1 \mathrm{~B}$ show the setup: Figure $1 \mathrm{~A}$ shows a drawing of a participant performing the matching task, and the four possible bar positions are marked. Figure 1B shows a picture of one of the bars and the protractor used to measure its orientation.

On each trial, one bar was placed on the left and the other on the right side of the body midline. Each participant stood in front of the whiteboard at a distance of about $30 \mathrm{~cm}$. The height of the whiteboard was adjusted for each participant so that the vertical distance
A

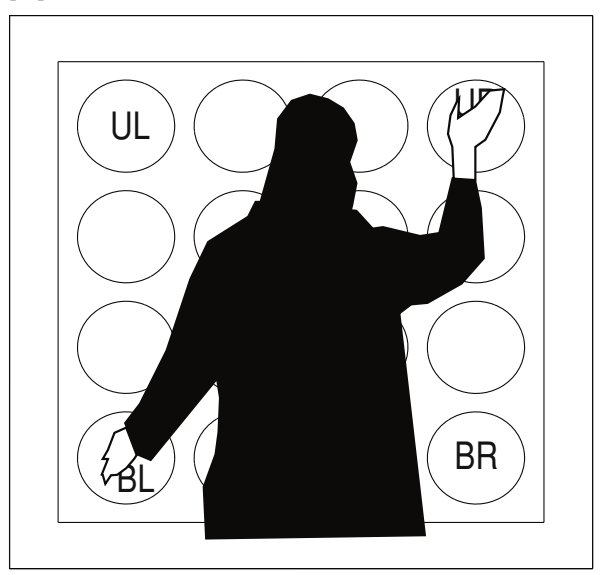

B

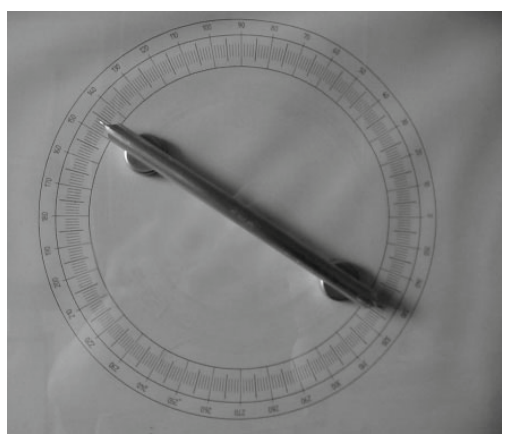

Figure 1. (A) A schematic of the setup in which participants performed the matching task. Bars could be presented on each of the four corners of a $4 \times 4$ grid. The letters in the plot indicate the four positions: upper left (UL), upper right (UR), bottom left (BL), and bottom right (BR). (B) A picture of one of the bars. The bar was attached to the whiteboard by means of magnets and could rotate around its center. After each trial, the experimenter measured the orientation of the bar by looking at the protractor printed on a sheet of paper attached to the whiteboard. 
between the upper bar positions and the participant's shoulder was $30 \mathrm{~cm}$. With the whiteboard in this position, all stimuli could be reached easily.

For the video recording of the participants' movements, a mini digital video camera (Aiptek DV3100+) was used. This camera produced AVI video files with a resolution of $320 \times 240$ pixels.

Design. Eight combinations of the locations of the two bars were used. One bar was presented to the right of the participant and one to the left. The following combinations were used (in the form "reference position-matching position," where UL denotes upper left, UR upper right, BL bottom left, and BR bottom right): UL-UR, UR-UL, UL-BR, BR-UL, BL-UR, UR-BL, BL-BR, and $\mathrm{BR}-\mathrm{BL}$. Each of these eight combinations was presented three times for each of the main orientations (horizontal $\left[0^{\circ}\right]$, vertical $\left[90^{\circ}\right]$, and two oblique orientations $\left[45^{\circ}\right.$ and $135^{\circ}$ counterclockwise with respect to horizontal]). The combination of all locations and orientations resulted in 96 trials per participant. The order of the trials was randomized across participants. To prevent the participants from simply matching a visualized orientation of $0^{\circ}, 45^{\circ}, 90^{\circ}$, or $135^{\circ}$, the main orientation, main orientation $-10^{\circ}$, and main orientation + $10^{\circ}$ were each presented once. For example, for the $45^{\circ}$ orientation, the orientations $45^{\circ}, 35^{\circ}$, and $55^{\circ}$ were used.

The 6 participants whose movements were video-recorded received only one set of 32 trials, which included only the four main orientations and the eight combinations of reference-bar and matchingbar locations.

Procedure. Before the start of the experiment, the experimental task was explained to each participant.

The experimenter displayed two pens on the table and rotated one so that its orientation matched the orientation of the other. The experimenter then explained that in the study, bars would be used instead of pens and that the task would be performed by touch instead of by sight. Following these instructions, the participant was blindfolded and guided to the whiteboard. The participants did not see the setup until after the experiment had concluded.

At the beginning of each trial, the reference and matching bars were positioned at the preselected locations, which were printed on a list. The experimenter then rotated the reference bar to the preselected orientation. The orientation of the matching bar was set in a random orientation that was at least $10^{\circ}$ off from the orientation of the reference bar. Before each trial, the experimenter told the participant which was the reference bar and which the bar to be rotated. If the participant rotated the incorrect bar, the reference bar was set to its original orientation and the trial was rerun. The participants were allowed as much time as they wanted for inspection of the reference bar and for setting the orientation of the matching bar. Typically, the participants took about $10 \mathrm{sec}$ per trial. They were asked to touch the bars with the inside of their hands and to stand upright without bending their knees. Bars on the left had to be touched with the left hand and bars on the right with the right hand. When the participants indicated their satisfaction with a setting, the experimenter determined the orientation of the matching bar by looking at the protractor on the whiteboard.

The experiment was divided into two blocks, and during a short break between the blocks the participants were guided to the other side of the room. This procedure allowed them to take off their blindfold during the breaks without seeing the setup.

The participants in the video-recorded version of the experiment performed all of their trials in one block, which took about $15 \mathrm{~min}$. The participants who were familiar with haptic matching experiments were told that any orientation could be presented and that it would be important to match the perceived orientation. The remaining 2 participants did not receive any information about the orientations used in the matching task.

The video recordings were analyzed after the experiment. From the video files, the final frames were extracted, just before each participant released the bars at the end of the trial. In these frames, the locations of the shoulder, the elbow, the hand, and the tip of the middle finger were determined visually by mouse clicks on the image of the frame. The locations were used to determine the orientations of the upper arm, the forearm, and the hand in space. For 2 of the participants, the hand and forearm orientations at the onset of the haptic inspection movements were measured in order to investigate whether either the initial hand and forearm or the final hand and forearm orientations affected the matching errors. From this analysis, it became clear that initial arm posture did not correlate with the matching errors, which led us to decide not to analyze those data for the remaining 4 participants.

\section{Results}

Participants made large errors in matching the orientation of the matching bar with that of the reference bar. Figure 2 shows the errors made by participant M.A. In this figure, the reference-bar orientations and positions are denoted by a thick bar, and the orientations produced by the participant are shown as thin lines at each of their respective positions. The other participants made errors in the same direction as those of M.A. Only the size of the errors differed across participants, with M.A. making relatively large errors. The errors in Figure 2 are shown with respect to their corresponding main orientation (either $0^{\circ}, 90^{\circ}, 45^{\circ}$, or $-45^{\circ}$ ). For example, if a participant matched a reference orientation of $55^{\circ}$ with an orientation of $70^{\circ}$, a line with an orientation of $70^{\circ}$ (matching bar) $55^{\circ}($ reference bar $)+45^{\circ}$ (main orientation $)=60^{\circ}$ would be shown.

Figure 2 shows that if the orientation of a bar on the right is matched with that of a bar on the left, its orientation is rotated clockwise (a positive deviation). If the orientation of a bar on the left is matched with that of a bar on the right, its orientation is rotated counterclockwise (a negative deviation). We used this fact when computing the mean signed errors. The mean signed errors were defined as the orientation of the left bar minus the orientation of the right bar, which resulted in mainly positive deviations. We could average the errors across matching direction, because the size of the errors did not differ with respect to matching direction (left to right vs. right to left).

The mean signed errors are shown in Figure 3. In this plot, means across participants and matching direction (from left to right and from right to left) are shown. The first two sets of bars show the horizontal and vertical orientations, and the last two sets show the oblique orientations. In an ANOVA, we tested the effects of stimulus position (8 levels) and orientation (4 levels). A complex pattern of results was found. The interaction effect was significant [Greenhouse-Geisser: $F(7.3,98)=5.078$, $p<.001]$, as was the main effect of location $[F(7,8)=$ $12.201, p<.01]$. No main effect of orientation was found $(p>.2)$. The analysis showed that overall the signed errors were significantly different from zero $[F(1,13)=$ $57.388, p<.001]$. Only one simple effect proved to be consistent across conditions: For each presented orientation, the errors were larger when the reference bar and the matching bar were presented at the bottom of the whiteboard (all $p$ values for each orientation <.05).

Kappers (2003) showed that systematic matching errors for the horizontal plane were participant dependent. At 

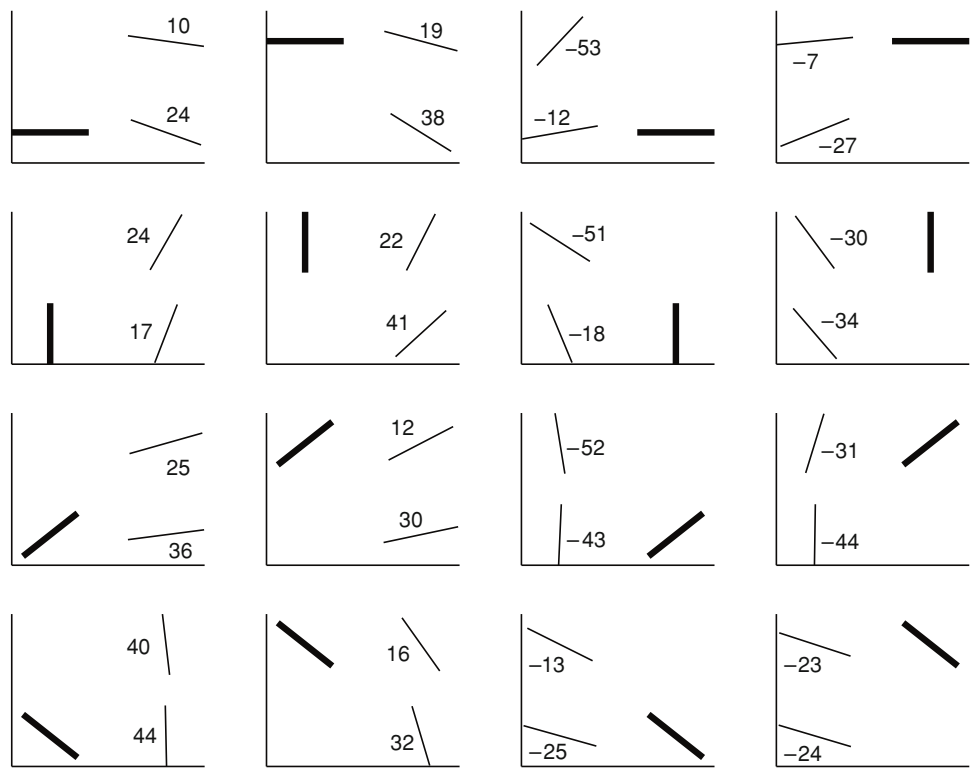

Figure 2. Signed errors of participant M.A. in Experiment 1. Two positions on the left and two on the right side could serve as positions for the reference or the matching bar. The thick lines show the presented main orientations of the reference bar. The two thin lines show the mean orientations produced by $M . A$. with respect to the main orientation at the two matching positions on the contralateral side. The numbers in the plot show the size of the mean signed error.

large stimulus distances, participants with relatively small errors showed a standard oblique effect on systematic errors: The mean signed errors were smaller for the horizontal and vertical orientations than for the oblique orientations. Participants with relatively large errors showed a reverse oblique effect in which they made smaller errors for the oblique orientations. We obtained the same results in our data for the frontoparallel plane. Figure 4 shows the size of the errors for the orthogonal and oblique orientations as a function of the mean error across all conditions.

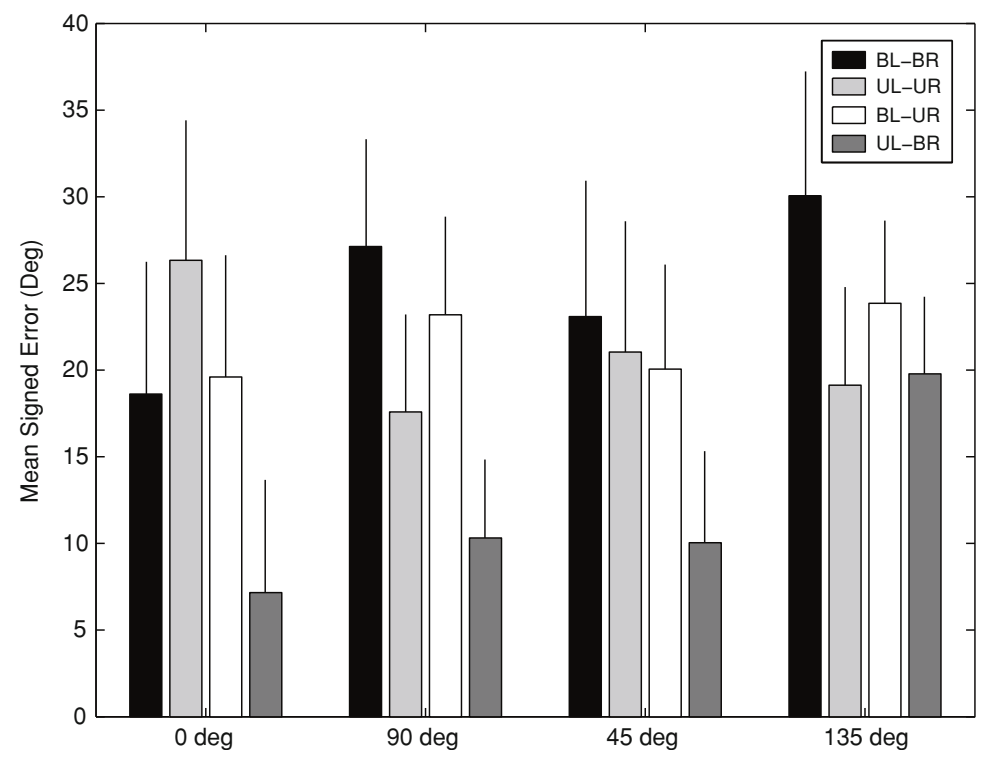

Figure 3. Mean signed errors per reference-bar orientation for each combination of the matching- and reference-bar locations in Experiment 1. Means across participants and matching directions (left to right and right to left) are shown. The lines on top of the bars show the $95 \%$ confidence intervals. 


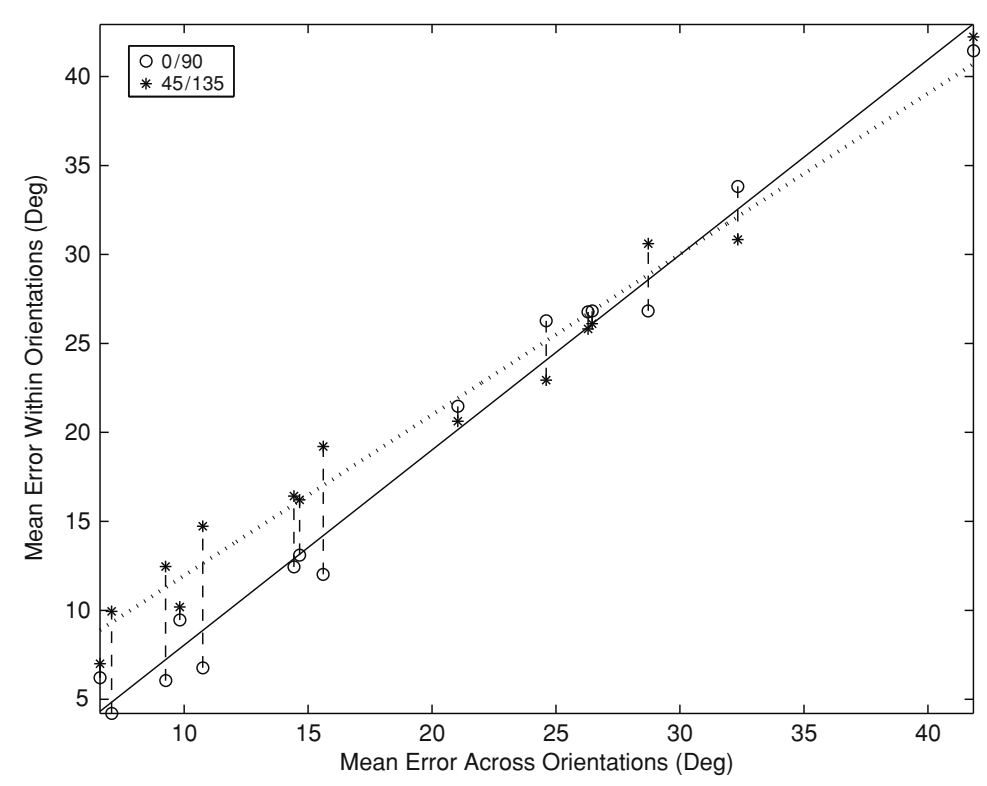

Figure 4. Mean signed errors in Experiment 1 per orientation $\left(0^{\circ}\right.$ and $90^{\circ}$ vs. $45^{\circ}$ and $135^{\circ}$ ) plotted against the mean overall error. Each pair of data points (connected by a dashed line) shows the data of 1 participant. Also included in the plot are the regression lines (a solid line for the $0^{\circ}$ and $90^{\circ}$ orientations and a dotted line for the $45^{\circ}$ and $135^{\circ}$ orientations).

Each pair of data points (connected by a dashed line) represents data from 1 participant. Two regression lines (the solid line represents the data for $0^{\circ}$ and $90^{\circ}$ and the dotted line those for $45^{\circ}$ and $135^{\circ}$ ) with different slopes and intercepts yield a significantly better fit of the data than does a single regression line $[F(2,10)=4.823, p=.05]$.

Kappers (2003) showed that the size of matching errors for the horizontal plane is gender dependent: Female par- ticipants made larger errors than do male participants. Figure 5 shows that a strong tendency toward this effect is also present in our data for the frontoparallel plane, which was confirmed by a one-sided $t$ test $[t(12)=1.749, p=.053]$.

Since 6 participants in our experiments were $\mathrm{PhD}$ students of the department who had heard about the systematic errors in haptic matching experiments before, we had the chance to test whether such knowledge has an effect

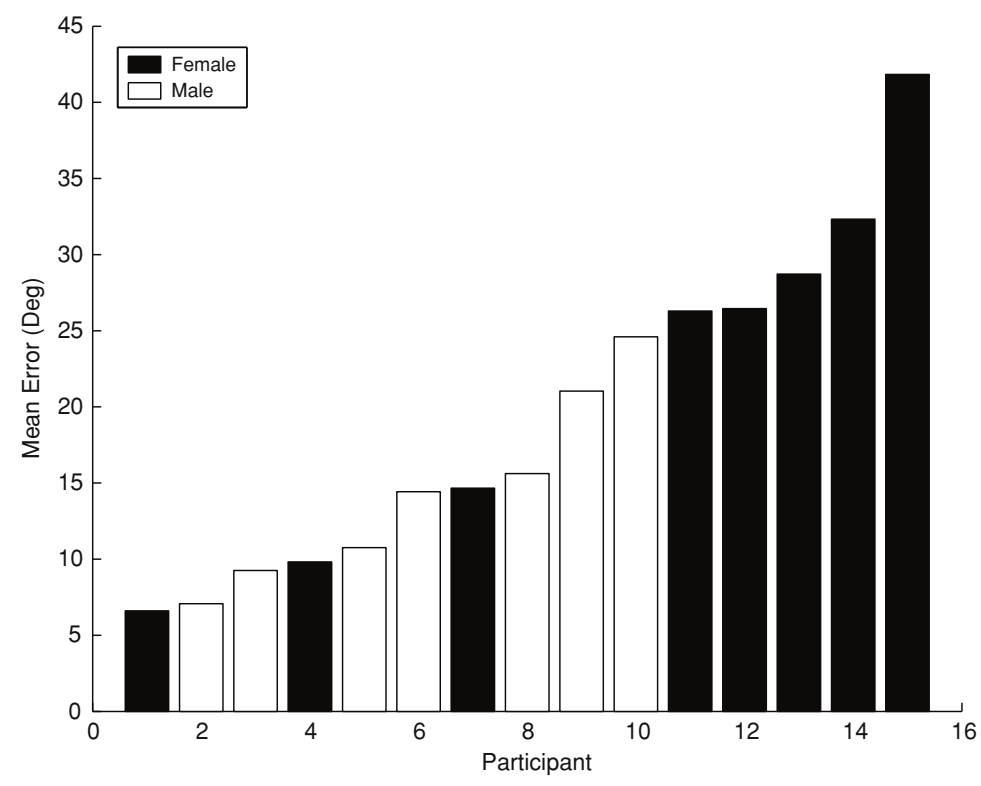

Figure 5. Mean signed errors per participant in Experiment 1. The data are sorted by size of the mean signed error. The solid bars show the mean errors for female participants, the open bars for male participants. 
on the size of errors. This did not appear to be the case: The systematic errors of the PhD students did not differ in size from those of the naive participants $[t(14)=0.367$, $p=.72]$.

For the 6 participants who were video-recorded while they performed 32 matching trials, we correlated the difference in hand orientations (left-hand - right-hand orientation) at the end of the matching movements with the signed error in the settings. No significant correlation was found across participants (mean $=-0.1457, p>$ .1). We also computed the correlation between the difference in forearm orientations and the size of signed errors, but again no substantial correlation was found (mean = $-0.1701, p>.1)$. An analysis of the forearm and hand orientation data of 2 participants at the beginning of the movements did not show higher correlations with the errors, and visual inspection of the hand and forearm orientations and the bar orientations did not reveal any other obvious relationship.

\section{Discussion}

Participants made large errors when haptically matching the orientation of a bar with that of a reference bar. These errors showed a specific pattern: The orientation of the matching bar on the right tended to be rotated clockwise in comparison with the correct orientation of the reference bar on the left. For the reference bars on the right, the opposite matching pattern was observed: The orientation of the matching bar on the left was rotated counterclockwise with respect to the reference orientation on the right. This pattern of results indicated that the haptic space used by the participants to perform the matching task was systematically deformed with respect to veridical.

We were able to replicate the reverse oblique effect found by Kappers (2003) for the horizontal plane. She found that for large distances between the matching bar and the reference bar, participants with relatively large matching errors tended to have larger errors for the horizontal and vertical orientations than for the oblique orientations. The fact that we could replicate this finding for the frontoparallel plane suggests that similar mechanisms underlie matching errors in both planes. Kappers (2003) showed that for stimuli at larger distances, hands were placed on the bars so that for oblique orientations, the stimuli were aligned with or perpendicular to the hand, whereas horizontal and vertical bar orientations had oblique orientations with respect to the hand. Participants with large deviations were biased by an egocentric frame, and an oblique effect in this egocentric reference frame would result in a reverse oblique effect in a physical reference frame.

For the midsagittal plane, Kappers (2002) showed that the vertical distance between the reference bar and the matching bar determined to a large extent the size of the matching errors. Our experimental design did not allow a similar comparison for the frontal plane. However, we found that the vertical positions of the two bars relative to the participant affected the size of the errors, which were larger when the reference and matching stimuli were pre- sented at the bottom of the whiteboard. This finding might be related to the fact that many participants reported that they found hand orientations for the bottom positions to be unnatural.

Kappers (2003) showed that female participants make larger matching errors than do male participants, and our findings for the frontoparallel plane agree with this result. The origin of this difference between female and male participants' performance is not known. A study by van Mier, Blommaert, and Kappers (2003) has shown that the difference in matching performance is already present at the age of 6 .

We were not able to quantitatively replicate Soechting and Flanders's (1993) and Kappers's (2003) interpretation that the matching errors are related to egocentric (forearm- or hand-related) coordinates. Our failure to find a relationship between forearm or hand orientation and matching errors might have been due to the limited number of positions of the reference and matching bars used in our study. Across trials with the same bar positions, hand and forearm orientations did not vary much, and the main part of the variation was due to bar position. Only eight combinations of reference- and matching-bar orientations were used, and these combinations were symmetrical in pairs. This small number of bar positions might have resulted in the small correlation between hand orientation and the size of the errors that we observed. Another possibility is that the failure to replicate the correlation between hand orientation and size of errors was due to the method for measuring hand orientation. In our experiment, we measured hand orientation while participants were performing the matching task; Kappers (2003) determined participants' hand orientation by asking them to put their hand at the bar position without actually performing the matching task. Soechting and Flanders varied arm posture by asking participants to match the reference bar at different positions in space. We could not quantitatively replicate the relationship between hand or forearm orientation and the size of matching errors. However, qualitatively, the direction of matching errors was consistent with Kappers's (2003) idea that parallelity judgments are made in a frame of reference intermediate between one fixed in space and an egocentric one.

\section{EXPERIMENT 2}

The results of Experiment 1 showed that participants made large systematic errors when asked to haptically match the orientation of a reference bar. These errors could originate in several stages of information processing between perceiving the reference bar and executing the movement to adjust the orientation of the matching bar. Experiments 2 and 3 were designed to determine which stage in the matching process might be the occasion for the errors. We assumed that participants first perceive the orientation of the reference bar and that this perceived orientation is then transferred to the location of the matching bar. Finally, the perceived and transferred reference-bar 
orientation is produced at the matching bar location. The video recordings of Experiment 1 showed that participants first rotated the matching bar across a large angle, followed by small corrections in which the orientations of both bars were extensively probed. This observation suggests that participants strongly rely on their perception of bar orientation. In Experiment 2, we tested the hypothesis that errors in matching the bars' orientations were due to errors in the perception of the reference orientation. We investigated haptic orientation perception by asking participants to verbally report the orientation of a bar at various locations in the frontoparallel plane. Participants were asked to imagine that the bar was the large hand of an analog clock attached to the whiteboard and to name the time on this imaginary clock.

\section{Method}

Participants. Twelve graduate or undergraduate students $(6$ male) took part in the experiment. Nine of them had participated in a haptic orientation-matching experiment at the department before, but none of them was familiar with the clock-naming task. Two of the participants (G.Y. and J.A.) were left handed; they performed the task with the same hand as the right-handed participants. The 5 participants who were not members of the Physics of Man Department were paid for their participation.

Design. For each participant, a list of randomly selected stimulus locations and bar orientations was generated. For each trial, the stimulus location was selected at random (either upper left, upper right, bottom left, or bottom right). The first 2 participants received randomly selected orientations between $0^{\circ}$ and $180^{\circ}$. After testing these 2 , we realized that the participants could only report angles in terms of an integer $\times 6^{\circ}$ (corresponding to the minutes on the clock). Therefore, the remaining 10 participants were presented with orientations corresponding to an integer number of minutes. On some of the trials, the participants were presented with a main orientation from Experiment 1, but only when this orientation was selected at random. Each participant carried out a total of 120 trials.

Procedure. Before blindfolding, the experimental task was explained to each participant visually. It was explained that they would be presented with bars in randomly selected orientations that they would then have to estimate. They were asked to imagine that the bar was the minute hand of a clock attached to the whiteboard. Their task was to name the time on that clock in minutes. The examples $0^{\circ}$ (horizontal), $90^{\circ}$ (vertical), and $42^{\circ}$ were shown by orienting a pen in those orientations and indicating the time: 15 past, on, and 7 past the hour, respectively. The participants were free to choose how they would report an orientation; most of them reported times between on the hour and half past, but some used times such as 45 past or 15 before. All reported clock times were later converted to orientations between $0^{\circ}$ and $180^{\circ}$

After the instructions, a participant was blindfolded and guided to the whiteboard. As in Experiment 1, they were positioned so that they stood at equal distances to the left and the right stimulus locations and at a distance of about $30 \mathrm{~cm}$ from the whiteboard. The height of the whiteboard was adjusted so the participant could easily reach each of the stimuli (so that, as before, a distance of about $30 \mathrm{~cm}$ was between the upper stimuli and the participant's shoulders). On each trial, the bar at the indicated location was rotated to match the orientation on the list generated for that participant.
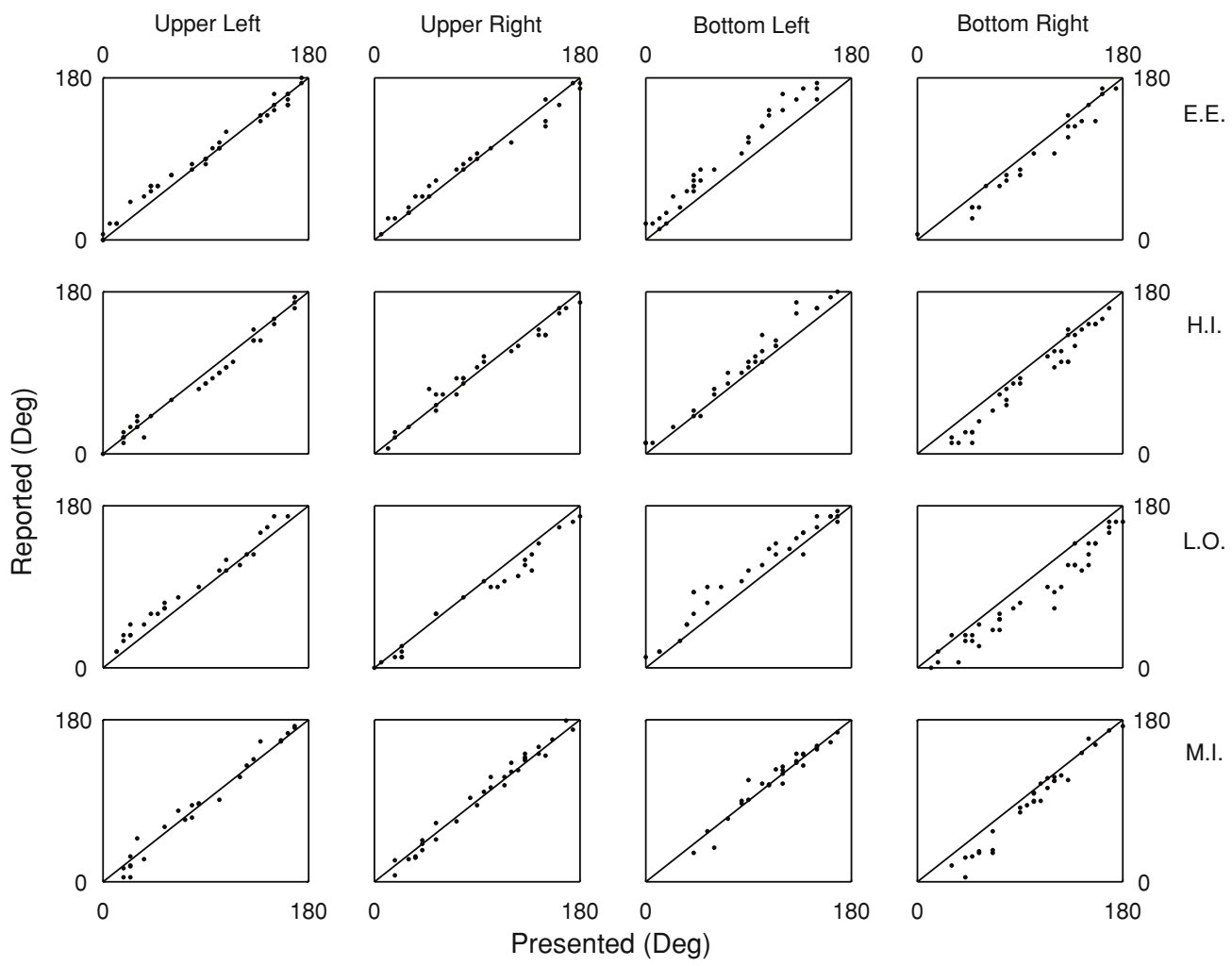

Figure 6. Presented and reported orientations for each bar location in Experiment 2. The data of 4 participants (E.E., H.I., L.O., and M.I.) are shown. Presented and reported orientations are expressed in terms of counterclockwise rotation with respect to the horizontal in degrees. 
The experimenter indicated to the participant which of the bar orientations was to be estimated by stating a position ("upper left," "upper right," "bottom left," or "bottom right"). The participants were asked to inspect bars on the right with the right hand and bars on the left with the left hand. The participant verbally reported the estimated orientation in terms of minutes, and the experimenter wrote down this number. The participants could take as much time as they wanted to haptically inspect the bar orientation; typically, they would inspect the bar for $15 \mathrm{sec}$.

The experiment was run in three sessions of 40 trials each, with short breaks in between. During these breaks, the participants were guided to the other side of the room, where they could take off the blindfold without seeing the setup.

\section{Results}

Figure 6 shows the presented and reported orientations of 4 participants (rows: E.E., H.I., L.O., and M.I.) at the four test locations (columns). Several participants showed a bias in their reports for certain orientations. For example, E.E. reported orientations for the bottom left bar that were generally smaller than the presented orientation. For this participant, this pattern reverses itself for the bottom right orientation. Participants H.I. and L.O. show the opposite pattern: They overestimated the orientations at the bottom left position and underestimated the orientations at the bottom right position.

Figure 7 shows the mean errors, with each bar showing the mean for 1 participant. Lines on top of the bars show $95 \%$ confidence intervals. Asterisks denote the $p$ value found in a $t$ test comparing the reported and presented orientations: A single asterisk denotes $p<.01$, and a double asterisk denotes $p<.001$. Although errors clearly differ across participants, a consistent pattern can be observed: Participants with an overestimation for the left positions show an underestimation for right positions, and vice versa. For the top stimulus locations, the correlation between errors on the left and on the right was $-.635(p<.05)$. For the bottom stimulus locations, a correlation between left and right errors of -.791 ( $p<$ .05 ) was found.

As in the matching task of Experiment 1, the errors were larger for the stimuli presented at the bottom than for those presented at the top of the whiteboard $(t=7.56$, $p<.001$ ).

Overall, the errors in this task were significantly smaller than those in the matching task of Experiment 1 $[F(1,16)=16.618, p<.001$; the data of the few participants who took part in both Experiments 1 and 2 were excluded from the ANOVA].

\section{Discussion}

The error patterns found in this clock-naming task differed substantially across participants. However, an interesting overall pattern was found: The errors within each

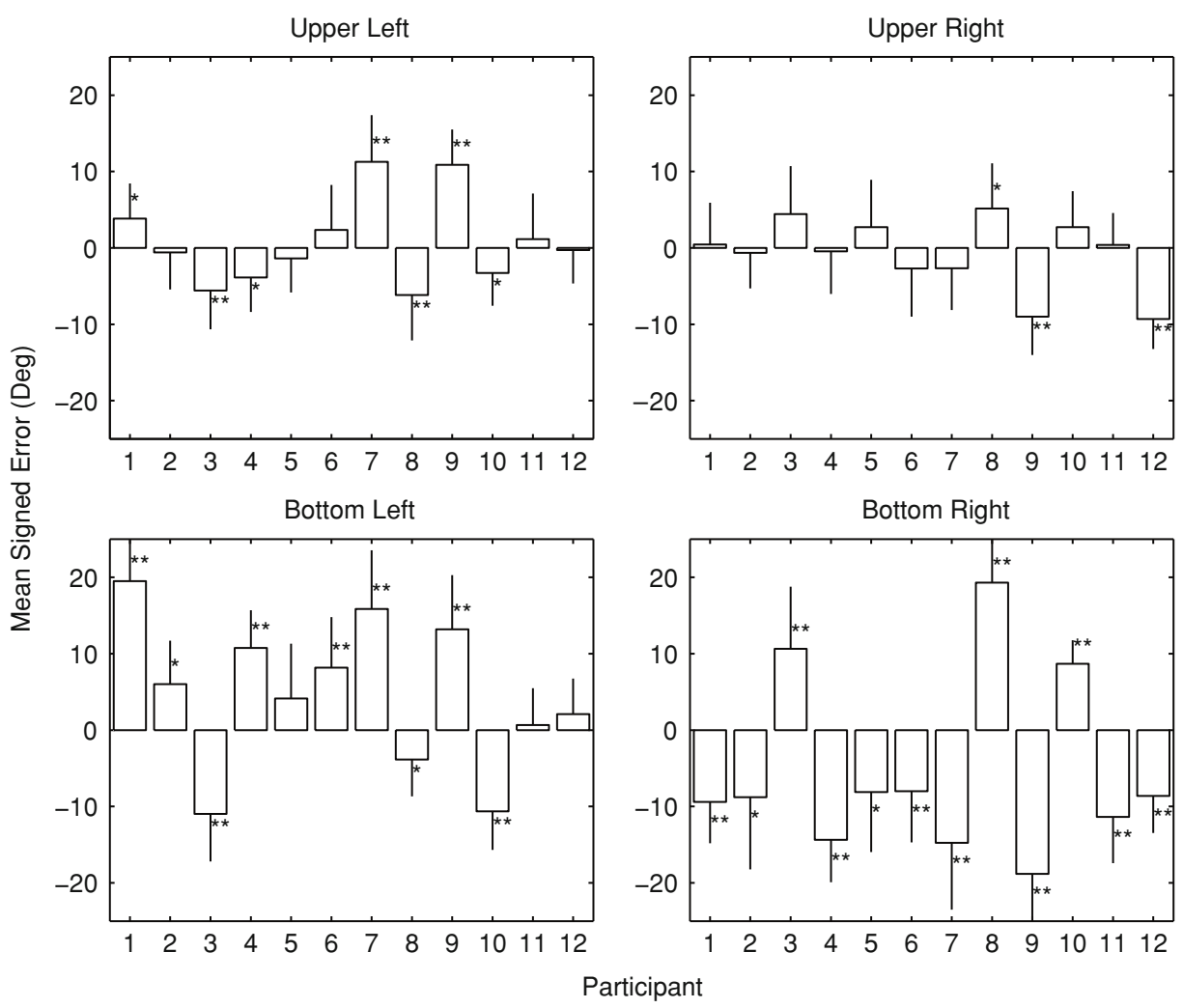

Figure 7. Mean error for each participant at each bar location in Experiment 2. Lines on top of the bars denote the $\mathbf{9 5 \%}$ confidence intervals. Asterisks denote the significance of the difference between the presented and the reported orientations. ${ }^{*} p<.01 .{ }^{* *} p<.001$. 
side (left or right) show a strong negative correlation. Our results extend earlier findings by Zuidhoek, Kappers, and Postma (2005), who found small systematic errors when participants were asked to verbally report the orientation of horizontally presented bars. They found that the size of the errors depended on the orientation of the hand.

Although many systematic errors were found within participants for the clock-naming task, the data of Experiment 2 show that the large systematic errors found in Experiment 1 cannot be due to errors in perception only, because the errors in the clock-naming task were significantly smaller than those in the haptic matching task. The absence of a significant overall mean error in Experiment 2 shows that the errors in Experiment 2 were also less consistent across participants than the errors in Experiment 1.

\section{EXPERIMENT 3}

In Experiment 2, we demonstrated that participants could name the orientation of haptically perceived bars without making large systematic errors. We therefore concluded that the large systematic errors found for the matching task of Experiment 1 were not due to an incorrect perception of the orientation of the reference bar. This conclusion leaves open the possibility that the large systematic errors for haptic matching were caused by an incorrect production of the perceived reference-bar orientation at the matching location. This possibility was tested in Experiment 3, in which we asked our participants to set bars in a verbally presented orientation. If production errors underlie the large matching errors of Experiment 1, large systematic errors should also be found for this orientation-setting task, and in addition, these errors should be consistent across participants and in the same direction as the matching errors.

\section{Method}

Participants. Nine participants (5 male) took part in the experiment. All were right handed. Six of them (physics undergraduate students at Utrecht University) were naive with respect to the purpose of the experiment and were paid for their participation. The other 3 participants (PhD students at Utrecht University) had taken part in a haptic matching experiment but had never performed a haptic production task before.

Design. The participants were asked to produce each of four orientations (which, as in the first experiment, were $0^{\circ}, 45^{\circ}, 90^{\circ}$, and $135^{\circ}$ ) with the four bars attached to the whiteboard. In contrast to Experiment 1, the participants were always asked to produce a main orientation. In Experiment 1, the main orientations $\pm 10^{\circ}$ were used to be sure that the participants were matching the presented orientation. We did not add this $10^{\circ}$ scatter to the instructed orientations in the production task of Experiment 3 because explaining the task to the participants would then have been more difficult. We wanted to make sure they understood the task properly. The participants were asked to set bars on the left with the left hand and bars on the right with the right hand. Each orientation was produced six times, and the order in which the orientations had to be produced was randomized for each participant.

Procedure. At the beginning of the experiment, the participants were blindfolded and guided to the whiteboard with the stimuli. This procedure prevented them from seeing the experimental setup until the end of the experiment.
A.B.

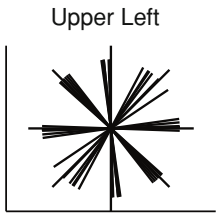

J.B.

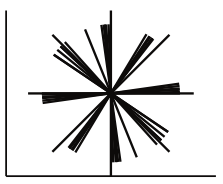

M.I.

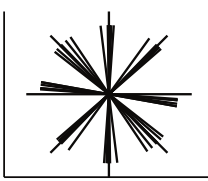

W.W.

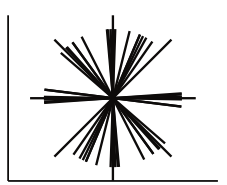

Upper Right
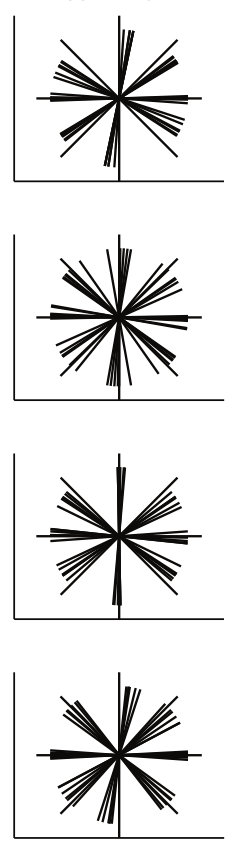

Bottom Left
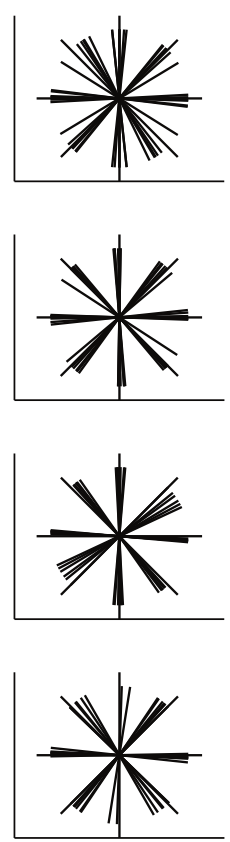

Bottom Right
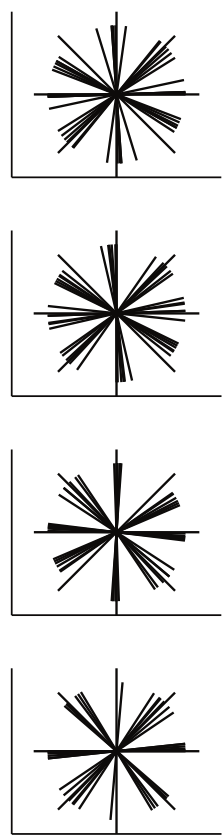

Figure 8. Orientations produced by 4 participants (A.B., J.B., M.I., and W.W.) in Experiment 3. The long lines show the instructed orientations, the short lines the produced orientations. Each column shows the settings at a different bar location. 
Before each trial, the experimenter set the bars in random orientations that were at least $10^{\circ}$ off from the orientation to be produced. Then the participant was told which orientation to produce. A possible instruction would be "set all four bars in a $45^{\circ}$ orientation," where $0^{\circ}$ would be horizontal and $90^{\circ}$ would be vertical. Following this instruction, the participant rotated all four bars on the whiteboard until they felt the bars to be in the instructed orientation. After releasing a bar, the participant was not allowed to change its orientation any more. While adjusting the orientation of a bar, a participant was not allowed to touch any other bar with the other hand. The participants could freely choose the order in which they set the orientations of the bars. As in Experiment 1, the experimenter determined the orientations of bars by looking at the protractors on the whiteboard.

\section{Results}

Participants could reproduce the requested orientations of the bars quite accurately. Figure 8 shows the settings of 4 participants (rows: A.B., J.B., M.I., and W.W.) for each of the bar positions (columns). In this figure, the long lines represent the four main orientations and the shorter lines show the participant's settings. All participants showed some systematic errors, but these errors were not consistent across participants. Figure 9 shows the mean errors for each participant. In the plot, mean errors are shown across orientations. The mean error across participants did not differ significantly from zero $(p>.10)$, indicating that the errors were not consistent across participants. No substantial correlation was found between the errors within the top or the bottom rows of stimuli (the respective correlations were $-.053, p>.10$, and $-.50, p>.10)$.

An oblique effect on the errors variable was present for the data in Experiment 3: $t$ tests on participant variances showed that across trials the participants set the horizontal and vertical bars more consistently than they did the oblique bars. For all four locations, the variance in the settings of horizontal and vertical orientations was less than the variance in the settings of oblique orientations (all four $p$ values $<.05$ ).

The errors in the production task were significantly smaller than those in the matching task of Experiment 1 $[F(1,20)=24.356, p<.001$; the data of the few participants who took part both in Experiment 1 and Experiment 3 were excluded from the ANOVA].

\section{Discussion}

Although some participants showed systematic errors when they haptically set the orientations of bars, there were no overall systematic errors across participants. The errors in the production task of Experiment 3 were much smaller than those found in the matching task of Experiment 1 , and this difference cannot be explained by the $10^{\circ}$ scatter that was added to the main orientations of Experiment 1 but not to the orientations of Experiment 3. For each of the experiments, we computed the error with re-

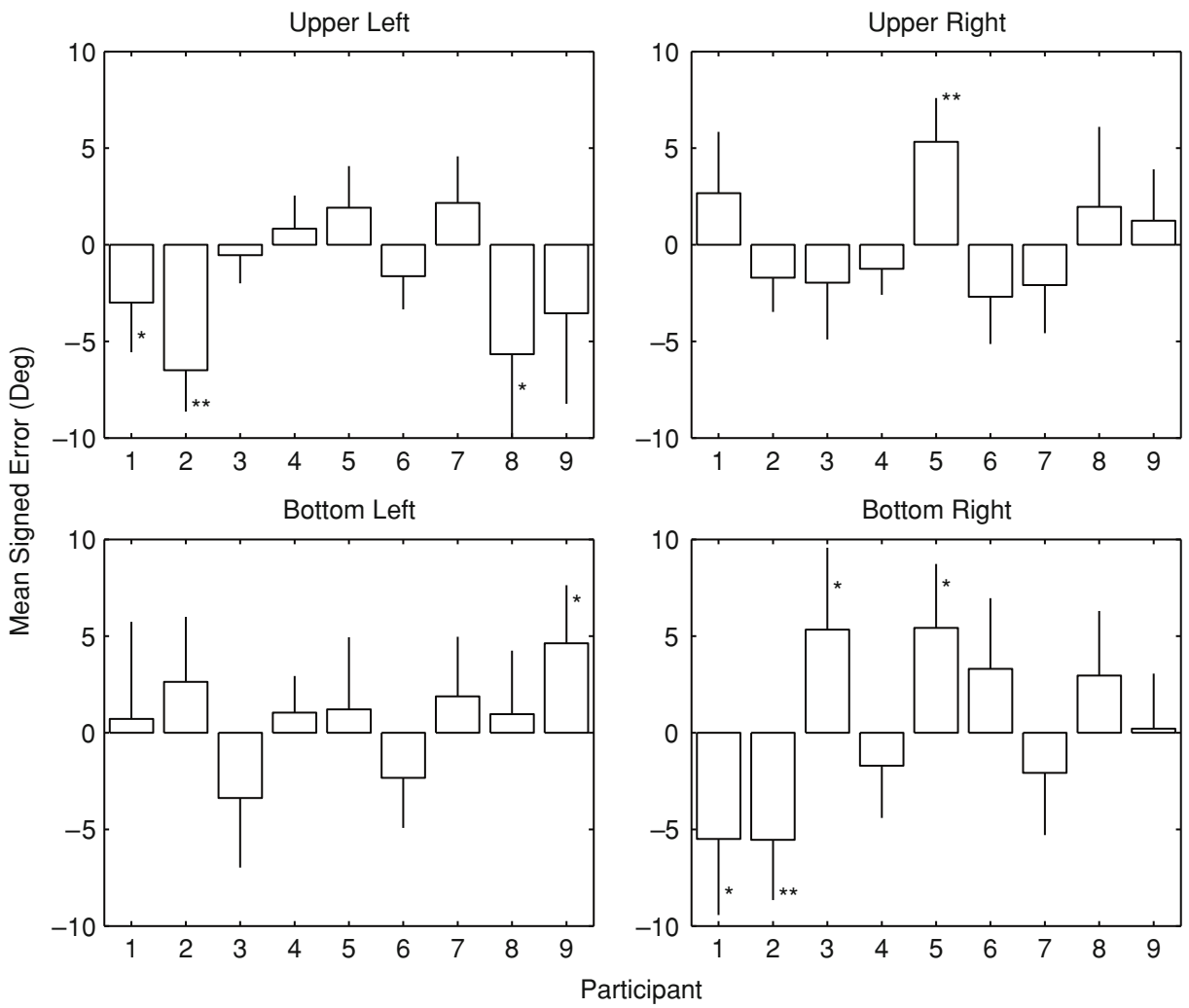

Figure 9. Mean signed errors for each participant at each bar location in Experiment 3. Lines on top of the bars denote the $\mathbf{9 5 \%}$ confidence intervals. Asterisks denote the significance of the difference between the requested and the produced orientations. ${ }^{*} p<.01 .{ }^{* *} p<.001$. 
spect to the reference orientation, which would probably lead to similar errors for similar reference orientations, unless large nonlinearities exist in the relation between reference orientations and matching errors. Similar errors for similar reference orientations were also expected on the basis of the results of Experiment 1, in which we found only small effects of the reference orientation.

Participants were less variable in their settings of horizontal and vertical orientations than of oblique orientations. These results relate to those of Luyat et al. (2001), who used a production task to determine the subjective vertical, which is defined as the perceived orientation of gravity during body or head rotation. They asked participants to produce each of four orientations $\left(-45^{\circ}, 0^{\circ}, 45^{\circ}\right.$, and $90^{\circ}$ ). When the participants' heads were in a vertical position, they did not make systematic errors, but oblique orientations were produced with greater variability. Gentaz, Badan, Luyat, and Touil (2002) asked healthy human participants and neglect patients to produce each of the four main orientations at each of two positions $(20 \mathrm{~cm}$ to the left or $20 \mathrm{~cm}$ to the right of the body midline). For the vertical and the $45^{\circ}$ oblique orientations, systematic errors were made that were absent for the horizontal and $-45^{\circ}$ orientations. The oblique orientations were also produced with greater variability than the horizontal and vertical orientations. The errors of the healthy young participants were independent of stimulus location. The results of Luyat et al. and Gentaz et al. correspond well with ours. The only differences concern the systematic errors Gentaz et al. found for the $45^{\circ}$ orientation and the vertical, for which they could give no explanation.

\section{GENERAL DISCUSSION}

In three experiments, we investigated the structure of frontoparallel space using three different tasks. In Experiment 1, participants were asked to match the orientation of two bars by rotating the matching bar so that it felt parallel to the reference bar. Participants performed the matching task for eight combinations of the positions of the reference and matching bars. In Experiment 2, we investigated the haptic perception of orientation by asking participants to tell the time if they assumed that the bar they felt was the large hand of a clock. Experiment 3 investigated orientation production through a task in which participants set bars at different positions in the frontoparallel plane in a verbally presented orientation. We hypothesized that if participants used the same underlying haptic structure for each task, errors would be similar across tasks.

In the haptic orientation-matching task of Experiment 1, participants made large systematic errors. Similar effects had been found for the horizontal plane and the sagittal plane (Kappers, 1999, 2002; Kappers \& Koenderink, 1999). For these two planes, large systematic matching errors were found, which were related to the orientations of the two hands (see Kappers, 2003, for an illustration of how this might work). Although we did not find a trial-by-trial correlation between hand orientation and the size of the matching errors in our matching experiments, we still think that hand orientation can, in part, explain the differences in errors across the eight combinations of reference and matching-bar positions.

Since we found large systematic errors in the frontoparallel plane similar to those found in the horizontal and sagittal planes, we wanted to take the investigation one step farther and investigate the origin of the errors. We hypothesized that the matching task involved three stages: (1) perception of the orientation of the two bars, (2) transfer of the perceived reference orientation to the matching location, and (3) production of the transferred orientation at the matching-bar location. In Experiments 2 and 3, we investigated two of the three stages separately-namely, perception (Experiment 2) and production (Experiment 3). In Experiment 2 (the perception task), we asked participants to report the orientation of the bars. They were asked to tell the time, assuming that the bar was the large hand of an analog clock. Although many systematic errors were found in the data, these errors were not consistent across participants. Also, the clock-naming errors were much smaller than the matching errors of Experiment 1 had been. In Experiment 3 (the production task), we asked participants to set bars at each of the four positions used in Experiment 1 in a predefined orientation. As with the perception task in Experiment 2, some systematic errors were observed for this bar-setting task. However, these systematic production errors were not consistent across participants, and they were much smaller than the matching errors of Experiment 1. A weighted sum of the errors in Experiments 2 and 3 could not explain the large systematic errors in Experiment 1, either, not even when only considering the repeated measures data of the few participants who took part in all three experiments. Note that the outcomes of these three experiments were contradictory: Participants could tell the orientation of the reference bar and could reproduce the orientation of that bar at the target orientation. However, when asked to perform perception and production in a single task, without explicitly naming the orientation of the reference bar, participants made large systematic errors.

The difference between the results of Experiment 1 and those of Experiments 2 and 3 can in part be explained by the difference between unimanual and bimanual matching. Experiment 1 made use of bimanual matching, but in Experiments 2 and 3 only one hand was used to perform the task. For example, Kappers (2002) found that haptic matching errors were smaller for unimanual matching, and a comparison of the data from Kappers and Koenderink (1999) and Kappers (1999) also leads to this conclusion. We think that the difference between unimanual and bimanual matching cannot account for the large differences between the errors of Experiment 1 and those of Experiments 2 and 3, however, because the difference in errors between unimanual and bimanual matching is simply too small. Moreover, although the difference in size of our errors is prominent, one should not ignore the fact that the patterns of the errors are also very different.

A comparison of the results in the matching task with those from the perception and production tasks suggests 
that the large systematic errors in the matching task might have originated in the transfer of the perceived bar orientation to the location of the matching bar, and not in the perception or production of the orientation. This transfer account would agree with the Riemannian space account mentioned in the introduction. One aspect of the theory of Riemannian spaces involves the transfer of objects with a certain orientation to a different position and the effects of the transfer on the orientation of the object.

An alternative explanation of the experimental results makes use of two different reference frames. In the production and perception tasks, participants might have been using a frame that was linked to the external, Euclidean space (allocentric space). The origin of this external space might be at the feet of the participants or at the corner of the whiteboard on which the stimuli were presented. One of the axes of the external space could be aligned with the perceived orientation of gravity, and the other two axes would be orthogonal to this axis. In the matching task, participants might have used an internal reference frame (egocentric space) that was related to the orientation of their forearm or hand (Kappers, 2003; Soechting \& Flanders, 1993). Earlier experimental results can also be understood in terms of these two different reference frames. In tasks in which participants simultaneously match the orientations of two bars (Kappers, 1999, 2002; Kappers \& Koenderink, 1999), a reference frame linked to the forearm or the hand is used, which results in large deviations from physical parallelity. If a delay is experienced, however, the external representation of orientation becomes more prominent, thus resulting in smaller matching errors (Zuidhoek et al., 2003). Newport et al. (2002) showed that haptic matching errors decrease when irrelevant visual information is available: Matching errors were smaller when the setup and the participant's hands were covered than when the participant was blindfolded. When participants have irrelevant visual information, they might rely to a larger extent on the external reference frame.

In our perception task, we asked participants to imagine that the bar was the large hand of a clock attached to the whiteboard. This task needs to be performed in an external reference frame, since the clock times that participants had to report were defined with respect to the reference frame of an external clock. In our orientation production task, participants were asked to produce orientations that also required the use of an external frame of reference. The data suggest, however, that in the matching task egocentric coordinates were used. Why participants consistently use this egocentric coordinate frame in the matching task is not known.

Our results can be explained in two ways: First, the transfer account states that errors originate from the transfer of the reference orientation to the matching location. The second account makes use of two spaces, an egocentric (body-related) one and an allocentric (Euclidean) one. This second account is in agreement with previous results and might therefore be favored, and the observed errors can then be understood as a weighted combination of the two spaces. The weights would depend on the task performed and could differ across participants.

Our results indicate that when performing tasks to investigate perceptual space, one needs to be cautious, since conclusions about the structure of the perceptual space might depend on the experimental task. Earlier, Cuijpers et al. (2003) also found that the induced structure of visual space is task dependent, by showing that the properties of visual space that could best describe their data were dependent on the experimental task.

\section{REFERENCES}

Appelle, S., \& Countryman, M. (1986). Eliminating the haptic oblique effect: Influence of scanning incongruity and prior knowledge of standards. Perception, 15, 325-329.

Appelle, S., \& Gravetter, F. (1985). Effect of modality-specific experience on visual and haptic judgment of orientation. Perception, 14, 763-773.

BLUMENFELD, W. (1937). The relationship between the optical and haptic construction of space. Acta Psychologica, 2, 125-174.

Coren, S. (1993). The left-hander syndrome. New York: Vintage.

CuiJpers, R. H., Kappers, A. M. L., \& Koenderink, J. J. (2002). Visual perception of collinearity. Perception \& Psychophysics, 64, 392-404.

Cuijpers, R. H., Kappers, A. M. L., \& KoenderinK, J. J. (2003). The metrics of visual and haptic space based on parallelity judgements. Journal of Mathematical Psychology, 47, 278-291.

Fukusima, S. S., Loomis, J. M., \& DA Silva, J. A. (1997). Visual perception of egocentric distance as assessed by triangulation. Journal of Experimental Psychology: Human Perception \& Performance, 23, 86-100.

Gentaz, E., Badan, M., Luyat, M., \& Touil, N. (2002). The manual haptic perception of orientations and the oblique effect in patients with left visuo-spatial neglect. NeuroReport, 13, 327-331.

GentaZ, E., \& HATwell, Y. (1995). The haptic "oblique effect" in children's and adults' perception of orientation. Perception, 24, 631-646.

Gentaz, E., \& Hatwell, Y. (1996). Role of gravitational cues in the haptic perception of orientation. Perception \& Psychophysics, 58, 1278-1292.

Gentaz, E., \& Hatwell, Y. (1999). Role of memorization conditions in the haptic processing of orientations and the "oblique effect." British Journal of Psychology, 90, 373-388.

Helmholtz, H. von (1962). Treatise on physiological optics (Vol. 3; J. P. C. Southall, Ed. \& Trans.). New York: Dover. (Original work published 1867)

Hillebrand, F. (1902). Theorie der scheinbaren Grösse bei binocularem Sehen [Theory of apparent size in binocular vision]. Denkschriften der Wiener Akademie (Mathematisch-Naturwissenschaft Klasse), 72, 255-307.

KAPPERS, A. M. L. (1999). Large systematic deviation in the haptic perception of parallelity. Perception, 28, 1001-1012.

KapPers, A. M. L. (2002). Haptic perception of parallelity in the midsagittal plane. Acta Psychologica, 109, 25-40.

Kappers, A. M. L. (2003). Large systematic deviations in a bimanual parallelity task: Further analysis of contributing factors. Acta Psychologica, 114, 131-145.

KAPPERS, A. M. L. (2004). The contributions of egocentric and allocentric reference frames in haptic spatial tasks. Acta Psychologica, 117, 333-340.

Kappers, A. M. L., \& KoenderinK, J. J. (1999). Haptic perception of spatial relations. Perception, 28, 1001-1012.

Koenderink, J. J., van Doorn, A. J., Kappers, A. M. L., \& Lappin, J. S. (2002). Large-scale visual frontoparallels under full-cue conditions. Perception, 31, 1467-1475.

Koenderink, J. J., van Doorn, A. J., Kappers, A. M. L., \& Todd, J. T. (2002). Pappus in optical space. Perception \& Psychophysics, 64, 380-391.

Lechelt, E. C., Eliuk, J., \& Tanne, G. (1976). Perception orientational 
asymmetries: A comparison of visual and haptic space. Perception $\&$ Psychophysics, 20, 463-469.

Lechelt, E. C., \& VerenKa, A. (1980). Spatial anisotropy in intramodal and cross-modal judgments of stimulus orientation: The stability of the oblique effect. Perception, 9, 581-589.

Luneburg, R. K. (1947). Mathematical analysis of binocular vision. Princeton, NJ: Princeton University Press.

Luyat, M., Gentaz, E., Corte, T. R., \& Guerraz, M. (2001). Reference frames and haptic perception of orientation: Body and head tilt effects on the oblique effect. Perception \& Psychophysics, 63, 541-554.

Meng, J. C., \& Sedgwick, H. A. (2001). Distance perception mediated through nested contact relations among surfaces. Perception $\&$ Psychophysics, 63, 1-15.

NewPort, R., RABB, B., \& JACKSON, S. R. (2002). Noninformative vision improves haptic spatial perception. Current Biology, 12, 1661-1664.

Soechting, J. F., \& Flanders, M. (1993). Parallel, independent channels for location and orientation in sensorimotor transformations for reaching and grasping. Journal of Neurophysiology, 70, 1137-1150.
Todd, J. T., Oomes, A. H. J., Koenderink, J. J., \& Kappers, A. M. L. (2001). On the affine structure of perceptual space. Psychological Science, 12, 191-196.

van Mier, H. I., Blommaert, J., \& Kappers, A. M. (2003). Haptic perception of parallelity in children and adults [Abstract, Program No. 196.18]. Society for Neuroscience 2003 Abstract Viewer/Itinerary Planner. Washington, DC: Society for Neuroscience. Available at sfn .scholarone.com/itin2003/index.html.

Wagner, M. (1985). The metric of visual space. Perception \& Psychophysics, 38, 483-495.

Zuidhoek, S., Kappers, A. M. L., \& Postma, A. (2005). Effects of hand orientation and delay on the verbal judgment of haptically perceived orientation. Perception, 34, 741-755.

Zuidhoek, S., Kappers, A. M. L., van der Lubbe, R. H. J., \& Postma, A. (2003). Delay improves performance on a haptic spatial matching task. Experimental Brain Research, 149, 320-330.

(Manuscript received August 20, 2004; revision accepted for publication March 16, 2005.) 